Experimental Facilities Division

Advanced Photon Source, Argonne National Laboratory

Argonne, IL 60439

A Simple Way of Characterizing X-ray Downwards-Deflecting Mirror-Bender Assemblies Using the Long Trace Profiler

Lahsen Assoufid and Peng Her

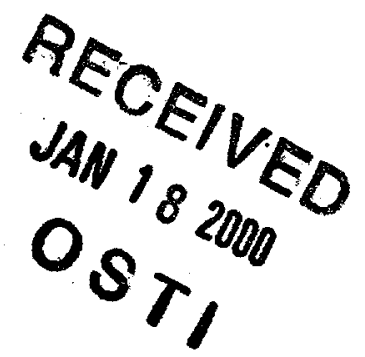

Conference Proceedings - American Institute of Physics

Synchrotron Radiation Instrumentation Conference

*This work is supported by the U.S. Department of Energy, Basic Energy Sciences-Materials Sciences, under contract \#W-31-109-ENG-38. 


\section{DISCLAIMER}

This report was prepared as an account of work sponsored by an agency of the United States Government. Neither the United States Government nor any agency thereof, nor any of their employees, make any warranty, express or implied, or assumes any legal liability or responsibility for the accuracy, completeness, or usefulness of any information, apparatus, product, or process disclosed, or represents that its use would not infringe privately owned rights. Reference herein to any specific commercial product, process, or service by trade name, trademark, manufacturer, or otherwise does not necessarily constitute or imply its endorsement, recommendation, or favoring by the United States Government or any agency thereof. The views and opinions of authors expressed herein do not necessarily state or reflect those of the United States Government or any agency thereof. 


\section{DISCLAIMER}

Portions of this document may be illegible in electronic image products. Images are produced from the best available original document. 


\title{
A Simple Way of Characterizing X-ray Downwards-Deflecting Mirror-Bender Assemblies Using the Long Trace Profiler
}

\author{
Lahsen Assoufid and Peng Her
}

Advanced Photon Source, Argonne National Laboratory, 9700 South Cass Avenue, Argonne, IL 60439

\begin{abstract}
A simple device composed of a modular double-pentaprism system that enables the long trace profiler (LTP) to measure mirrors in nonconventional ways, i.e., in the verticaldownward and sideways positions, has been devised and implemented in the Advanced Photon Source (APS) long trace profiler (LTP II). The systems is very useful in calibrating mirrorbender assemblies. This paper describes the system and gives results of measurements performed with it on a mirror used at the APS.
\end{abstract}

\section{INTRODUCTION}

The long trace profiler (LTP) is an optical profilometer originally developed by P.Takacs et al. (1) to measure the surface figure error and curvature of mirrors used to reflect x-rays in synchrotron radiation beamlines. The LTP system in use at the APS, LTP II, incorporates a number of features that considerably improved its measurement repeatability and accuracy (2). In its standard configuration, the LTP probe beam is oriented vertically and the surface under test has to be measured with its reflecting surface facing upwards. However, in many cases, mirrors used in synchrotron radiation beamlines are set up to deflect the incident x-ray beam either in the sideways or in the downwards-deflecting positions. This occurs, for example, in cases where a mirror is used for beamline branching or when a second mirror is used to vertically deflect or focus the incident x-ray beam. Therefore, these mirrors need to be characterized in their operating position in order to get their true figure error. Moreover, when integrated into a bending mechanism, their bender needs to be calibrated before installation in the beamline.

To characterize mirrors in the horizontally deflecting position, the LTP II probe beam needs to be deflected by a 90-degre angle to be perpendicular to the surface of the mirror under test, and when characterizing a mirror deflecting downwards, the LTP II probe beam needs to be bent an additional 90 degrees upwards. There are two simple ways of doing this (without affecting the original LTP design). One way is to use simple flat deflecting mirrors, the other is to use pentaprisms. Pentaprisms were chosen because flat mirrors have the disadvantage of being difficult to align, while pentaprisms are insensitive to tilt as a rigid body in the plane of refraction, and, therefore, their alignment is less critical. In the following, the system is described, possible related sources of systematic errors are discussed, and an example of measurements with it is presented.

\section{DESCRIPTION OF THE SYSTEM}

Figure 1 shows a photograph of the system. The test beam emanating from the LTP's optical board is bent by the first pentaprism (PP\#1) to a 90-degree angle to become 
horizontal; this position can be used to characterize mirrors and mirror-bender assemblies that are mounted to reflect an x-ray beam in the horizontal direction, or, simply, when measurements without the effect of gravity are desired. The second pentaprism (PP\#2) bends the probe beam an additional 90 degrees upwards and is added to characterize mirrors mounted with their reflecting surface facing downwards.

The system was built using mainly standard modular Microbench ${ }^{\mathrm{TM}}$ structural mounting components from Spindler \& Hoyer. The system is attached to the base of the LTP II's optical board. The pentaprisms can be adjusted independently in the vertical and the horizontal directions by simply sliding the vertical or horizontal rods, thus allowing one to scan any profile line on the surface of the mirror under test.

\section{PERFORMANCE TESTS}

The system was tested on a 364-mm long cylindrical silicon substrate with a radius of curvature of 56 meters. In order to avoid an edge effect, the scanned profile was limited to the 296-mm central length. The table below compares measurements performed on the mirror facing upwards and downwards.

\begin{tabular}{lccc}
\hline $\begin{array}{l}\text { Mirror } \\
\text { position }\end{array}$ & $\begin{array}{c}\text { Det0-slope error } \\
(\mu \mathrm{rad}), \text { rms }\end{array}$ & $\begin{array}{c}\text { Det1-slope error } \\
(\boldsymbol{\mu r a d}), \text { rms }\end{array}$ & $\begin{array}{c}\text { Radius } \\
\text { (meter) }\end{array}$ \\
\hline Face up & 1521.4 & 1.75 & 56.35 \\
Face down & 1521.4 & 2.20 & 56.16 \\
\hline
\end{tabular}

In each case, 10 profiles are averaged. Det0-slope error corresponds to the root mean square (rms) slope error obtained when only the tilt is removed from the measured profiles, while det1-slope error represents the rms residual slope error values obtained after a best-fit radius was applied to the det 0 profiles.

The difference in the measured radii is only 0.19 meter (or about $0.34 \%$ ) while that in the residual slope error is $0.45 \mu \mathrm{rad}$ (or about $26 \%$ ) which is, a priori, within the LTP accuracy. However, remember, additional optical elements are added to LTP II optical system and, therefore, can contribute to system's systematic errors (see the following section.)

\section{SOURCES OF SYSTEMATIC ERRORS}

As the mirror is scanned, the LTP II's laser probe beam is likely to walk across the faces of the pentaprims. Therefore, any imperfection due to inhomogeneity or surface figure error in the pentaprism will be interpreted as the mirror surface slope. This occurs particularly when large slope variations are encountered as is the case for mirrors shaped or bent to tight radii of curvature or mirrors with large figure errors. This beam-walk can also be generated by the pitch and the yaw of the LTP optical board during its translation. This situation has been encountered by Takacs and his co-workers in the vertical scanning long trace profiler VSLTP (3), in which the optical board is stationary and a pentaprism is used to direct to probe beam to the measurement point. The contribution to their measurements was found to be in the order of $0.5 \mu \mathrm{rad}$ (4), which is about the same magnitude as our value quoted in section 3 .

In the LTP II, these errors can be further exacerbated by the mirror-pentaprism distance as the return point of the probe beam on the pentaprism surface deviates further from its origin with increasing arm length. 
Other sources of errors that have be accounted for are a) the air temperature gradient, b) the mounting strain, and c) the cleanness of the optical components, particularly the faces of the pentaprisms. The air temperature gradient will cause a spurious tilting or bending of the laser probe beams thus resulting in surface slope error. Fingerprint marks were inadvertently left on one of the pentaprism and were found to increase the measured residual rms slope error by more than a factor of two.

\section{EXAMPLE OF MEASUREMENTS}

Before installing a mirror-bender assembly in a beamline it is very important to know the mirror's initial position and shape and its bender's calibration curve. This LTP system has been very useful in providing such data to the APS users. As an example, we give results of measurements for a vertically focusing mirror-bender assembly used in sector 18 at the APS. The mirror is deflecting downwards and was supported by a series of tension springs to compensate for gravity (5). The mirror was measured (facing upwards) before being mounted into the bender. After the mounting, the tension springs were tuned individually to set the mirror in a flat position, and the LTP II was used to monitor the mirror's shape. Figure 2 shows a plot of the mirror slope error profiles at various stages of the bender position from totally relaxed (i.e., no springs attached) to a flat position (home position after adjustment of the tension springs was completed).

\section{CONCLUSIONS}

The described device was useful in calibrating several APS beamline mirror-bender assemblies that have their reflecting surface facing downwards. The scheme has the disadvantage of adding more optical elements to the LTP optics board. Therefore, to achieve accurate measurements high-quality pentaprisms are required, along with excellent temperature control.

\section{ACKNOWLEDGMENTS}

L.A. would like to thank Shinan Qian (at BNL) and Steven Irick (at ALS) for useful discussions. This work is supported by the U. S. Department of Energy, BES-Materials Sciences, under contract No. W-31-109-ENG-38.

\section{REFERENCES}

1. P. Z. Takacs, S.-C. K. Feng, E. Church, S.-N. Qian, and W.-M. Liu, "Long trace profiler measurements on cylindrical aspheres," Proc. SPIE 966, (1989), pp. 354-364.

2. Peter Z. Takacs, Eugene Church L. church, Cynthia Bresloff, and Lahsen Assoufid, "Improvement in the accuracy and the repeatability of long trace profiler measurements," Applied Optics 38 (25) (1999), pp.5468-5479.

3. Haizhang Li, Xiaodan Li, Manfred W. Grindel, and Peter Z. Takacs, "Measurement of x-ray telescope using a vertical scanning long trace profile," Opt. Eng. 35(2) (1996), pp. 330-338.

4. P. Z. Takacs, private communication.

5. This mirror was measured for Robert Fischetti \& co-workers at Bio-CAT in sector 18, Advanced Photon Source, Argonne National Laboratory, Argonne, Illinois. 


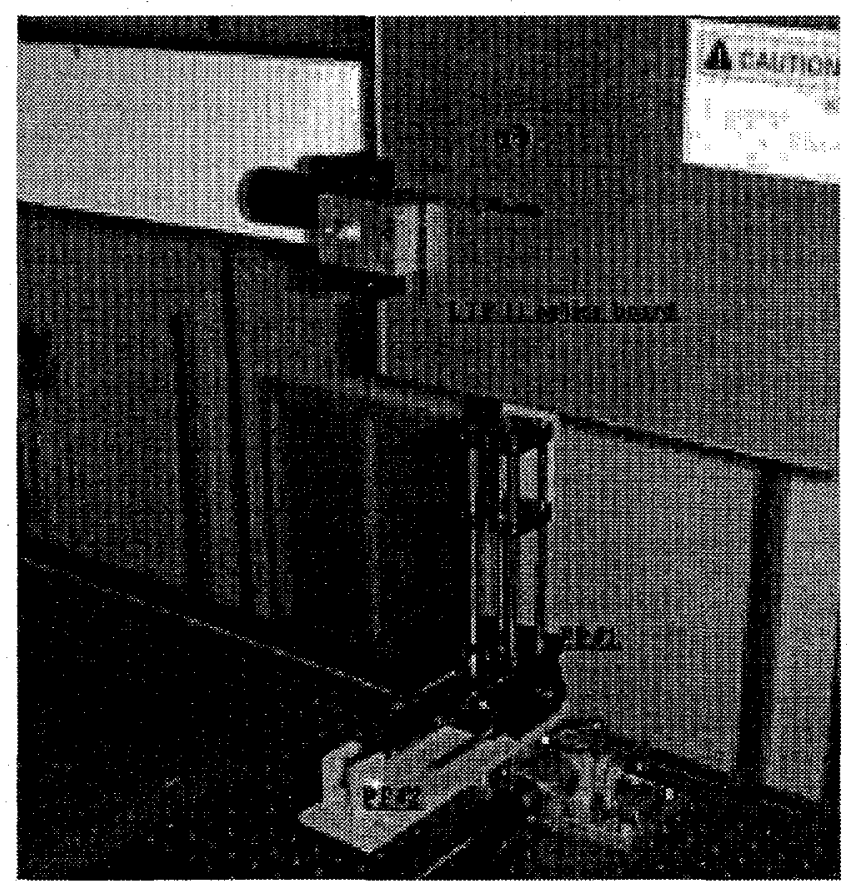

Figure 1. Photograph of the LTP II optical board with the scanning device mounted at the bottom. Pentaprism PP\#1 deflects the LTP I's laser probe beam at 90-degree angle and is used to evaluate mirrors facing sideways. Penatarism PP\#2 deflects the probe beam an additional 90 degrees upwards in order to measure mirrors and mirror-benders mounted to deflect the incident $\mathrm{x}$-ray beam downwards. The pentaprisms can be adjusted to scan any line on the surface of the test mirror under.

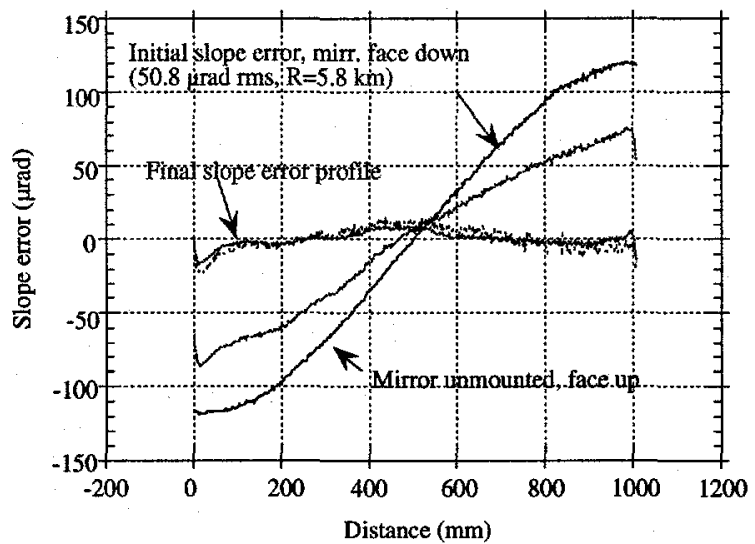

Figure 2. Slope error profiles for a vertically focusing mirror used in sector 18 at the APS. The mirror's reflecting surface faces downwards, and its bender was calibrated using the system shown in Figure 1. The plots represent slope error profiles at various positions of the bending mechanism. 